\title{
FINITE ELEMENT COMPUTATIONS OF KOLMOGOROV-PETROVSKY-PISKUNOV FRONT SPEEDS IN RANDOM SHEAR FLOWS IN CYLINDERS*
}

\author{
LIHUA SHEN ${ }^{\dagger}$, JACK XIN $^{\ddagger}$, AND AIHUI ZHOU ${ }^{\S}$
}

\begin{abstract}
We study the Kolmogorov-Petrovsky-Piskunov minimal front speeds in spatially random shear flows in cylinders of various cross sections based on the variational principle and an associated elliptic eigenvalue problem. We compare a standard finite element method and a two-scale finite element method in random front speed computations. The two-scale method iterates solutions between coarse and fine meshes and reduces the cost of the eigenvalue computation to that of a boundary value problem while maintaining the accuracy. The two-scale method saves computing time and provides accurate enough solutions. In the case of square and elliptical cross sections, our simulation shows that larger aspect ratios of domain cross sections increase the average front speeds in agreement with an asymptotic theory.
\end{abstract}

Key words. Kolmogorov-Petrovsky-Piskunov front speeds, random shear flows in cylinders, stochastic eigenvalue problems, two-scale finite element method

AMS subject classifications. 65C $30,65 \mathrm{C} 05,65 \mathrm{~N} 30,65 \mathrm{~N} 25$

DOI. $10.1137 / 080717420$

1. Introduction. Front propagation in heterogeneous flows is an active research area in applied science and mathematics $[7,14,15,18,19,25,32,33,36]$. A fundamental problem is to characterize and compute large-scale front speeds in random flows [10, 19, 30, 31, 39]. The Kolmogorov-Petrovsky-Piskunov (KPP) minimal front speeds admit a variational characterization in terms of the principal eigenvalue or principal Lyapunov exponent of an associated linear operator [6, 5, 16, 37, 28, 29]. The variational principle of KPP front speeds makes accurate and efficient analytical and numerical studies possible. It is known that KPP front speeds are enhanced by spatially random shear flows in cylinders, with a quadratic (linear) law in the small (large) root mean square amplitude regime; see [28] and the references therein. However, less is known about how the domains influence the front speeds.

In this paper, we shall study the dependence of KPP front speeds (in spatially random shear flows) on the aspect ratio of cylindrical cross sections. We shall use both numerical methods and asymptotic methods. Let $D \equiv \mathbb{R} \times \Omega$, where $\Omega \subset \mathbb{R}^{2}$ is a simply connected bounded domain with a Lipschitz continuous boundary. The reactive scalar equation is

$$
u_{t}=\Delta_{x, y} u+B \cdot \nabla_{x, y} u+f(u)
$$

${ }^{*}$ Received by the editors March 2, 2008; accepted for publication (in revised form) July 18, 2008; published electronically November 12, 2008. This work was partially supported by the National Science Foundation (NSF) of China under grant 10425105, the National Basic Research Program under grant 2005CB321704, and US NSF grant DMS-0549215.

http://www.siam.org/journals/mms/7-3/71742.html

${ }^{\dagger}$ Department of Mathematics, Capital Normal University, Beijing 100037, China (shenlh@lsec.cc. ac.cn).

${ }^{\ddagger}$ Department of Mathematics, University of California at Irvine, Irvine, CA 92697 (jxin@math. uci.edu).

$\S$ LSEC, Institute of Computational Mathematics and Scientific/Engineering Computing, Academy of Mathematics and Systems Science, Chinese Academy of Sciences, P.O. Box 2719, Beijing 100080, China (azhou@lsec.cc.ac.cn). 
where $f(u)=u(1-u)$, the KPP nonlinearity; $x \in \mathbb{R}, y=\left(y_{1}, y_{2}\right) \in \Omega \subset \mathbb{R}^{2}$; $B=(b(y, \omega), \overrightarrow{0}), b(y, \omega)$ being a stationary Gaussian process with mean zero; and parameter $\omega$ refers to a random sample or realization. The zero Neumann boundary condition is imposed on $u$ at $\partial \Omega$. The KPP minimal speed along $x$ is given by $[6,5,36,26]$

$$
c^{*}=c^{*}(\omega)=\inf _{\lambda>0} \frac{\mu(\lambda, \omega)}{\lambda},
$$

where $\mu$ is the principal eigenvalue with a positive eigenfunction of the eigenvalue problem

$$
\left\{\begin{aligned}
\Delta \phi+\left[\lambda^{2}+\lambda b(y, \omega)+f^{\prime}(0)\right] \phi & =\mu(\lambda, \omega) \phi \text { in } \Omega, \\
\frac{\partial \phi}{\partial \nu} & =0 \text { on } \partial \Omega .
\end{aligned}\right.
$$

The KPP front speed computation becomes that of a principal eigenvalue problem in a general domain $\Omega$. We shall perform Monte Carlo simulation of the KPP minimal front speed ensemble by solving a large number of eigenvalue problems (1.3) and then minimizing the objective function (1.2). The shear flow $b$ will be random but smooth, and its spectral energy decays rapidly towards high frequencies. A resolved computation is feasible with refined finite element meshes. To reduce the computational cost in solving the stochastic eigenvalue problem (1.3), we employ a so-called two-scale finite element method, first proposed in [23] and later developed in $[8,9,11,20,24,34,38]$. The two-scale method reduces the cost of eigenvalue computation to the level of computing a boundary value problem. The method is iterative and related to $[22,35]$.

Our method is formulated for KPP reactions. For other nonlinearities, the variational formula (1.2) provides an upper bound only. However, it is known in many cases (including fronts through shear flows) that qualitative properties of front speeds of non-KPP reactions are the same as the KPP ones; see [15, 28, 36, 40] among others. As of now, computing the non-KPP random front speeds relies on direct simulation of the governing equation (1.1) in time [28]. Some non-KPP front speeds satisfy minmax principles [17, 18]. Though helpful for analysis of random front speeds [27], the min-max principles have not been utilized for computation.

This paper is organized as follows. In section 2, we present standard and twoscale finite element methods and their convergence properties. We also derive an asymptotic theory of KPP minimal speeds in the limit of thin domains, implying that larger aspect ratios of domains lead to larger front speeds. In section 3, we show numerical results of random KPP front speeds in rectangular and elliptical domains of various aspect ratios. The front speeds always increase with the aspect ratio of domains, in agreement with the asymptotic theory. We also compute probability distributions of random front speed and compare costs as well as the efficiency of the standard and two-scale methods. Concluding remarks are in section 4.

\section{Numerical methods and asymptotic theory.}

2.1. Preliminaries. We use the standard notation for Sobolev space $W^{s, p}(\Omega)$ and their associated norms and seminorms $[1,12]$. For $p=2$, we denote $H^{s}(\Omega)=$ $W^{s, 2}(\Omega),\|\cdot\|_{s, \Omega}=\|\cdot\|_{s, 2, \Omega}$, and $\|\cdot\|_{\Omega}=\|\cdot\|_{0,2, \Omega}$. Let $(\cdot, \cdot)$ be the standard inner product of $L^{2}(\Omega)$. Throughout this paper, the letter $C$ (with or without subscripts) denotes a positive (random) constant independent of mesh sizes. 
Consider the following problem: Find $\phi \in H^{1}(\Omega)$ and $\mu \in \mathbb{R}$ such that

$$
\left\{\begin{aligned}
\Delta \phi+V(y, \omega) \phi & =\mu(\omega) \phi, \quad y \text { in } \Omega \\
\frac{\partial \phi}{\partial \nu} & =0, \quad y \in \partial \Omega
\end{aligned}\right.
$$

where $V(y, \omega)$ is a stationary continuous scalar random process in $y$ and so $V(y, \omega) \in$ $L^{\infty}(\Omega)$. Let $\mu \equiv \mu(\omega)$ be the principal (simple) eigenvalue with corresponding eigenfunction $\phi>0$ and $\|\phi\|_{0, \Omega}=1$.

Define $a(\phi, v)=\int_{\Omega} \nabla \phi \nabla v-\int_{\Omega} V(y, \omega) \phi v$; the variational form for (2.1) is

$$
a(\phi, v)=-\mu(\omega)(\phi, v) \quad \forall v \in H^{1}(\Omega) .
$$

Note that if we define

$$
\tilde{a}(\phi, v)=a(\phi, v)+\xi(\phi, v)
$$

for some constant $\xi>0$, then (2.2) is equivalent to

$$
\tilde{a}(\phi, v)=\tilde{\mu}(\phi, v) \quad \forall v \in H^{1}(\Omega),
$$

where $\tilde{\mu}=-\mu+\xi$. Select a positive random constant $\xi$ so that $\tilde{a}(\phi, v)$ is a positive symmetric definite bilinear form and satisfies

$$
C^{-1}\|u\|_{1, \Omega}^{2} \leq \tilde{a}(u, u) \quad \forall u \in H_{0}^{1}(\Omega) .
$$

We compute the principal eigenvalue $\mu$ of (2.2) or, equivalently, the minimal eigenvalue of (2.3). We shall use the following property of eigenvalue and eigenfunction approximations $[3,4,38]$.

Proposition 2.1. Let $(\tilde{\mu}, \phi)$ be an eigenpair of (2.3). For any $w \in H^{1}(\Omega) \backslash\{0\}$, there holds

$$
\frac{\tilde{a}(w, w)}{(w, w)}-\tilde{\mu}=\frac{\tilde{a}(w-\phi, w-\phi)}{(w, w)}-\tilde{\mu} \frac{(w-\phi, w-\phi)}{(w, w)} .
$$

Let $T^{h}(\Omega)$, consisting of shape-regular simplices, be a finite element mesh over $\Omega$ with mesh size $h=\max _{x \in \Omega} h(x)$, where the function $h(x)$ denotes the diameter of the element $\tau$ containing $x$. Define $S^{h}(\Omega)$ to be the space of continuous functions on $\Omega$ such that for $v \in S^{h}(\Omega), v$ restricted to each $\tau$ is linear, namely,

$$
S^{h}(\Omega)=\left\{v \in C(\bar{\Omega}):\left.v\right|_{\tau} \text { is linear } \forall \tau \in T^{h}(\Omega)\right\} .
$$

We refer the reader to $[12,13]$ for its basic properties. For instance, for a given $u \in H^{1+s}(\Omega)$, there holds

$$
\inf _{v \in S^{h}(\Omega)}\left(h^{-1}\|(u-v)\|_{0, \Omega}+\|u-v\|_{1, \Omega}\right) \leq C h^{s}\|u\|_{1+s, \Omega}, \quad 0 \leq s \leq 1 .
$$

2.2. One-scale discretization scheme. The standard finite element discretization for (2.2) is a one-scale discretization: Find $\phi_{h} \in S^{h}(\Omega)$ and $\mu_{h} \in \mathbb{R}$ such that $\left\|\phi_{h}\right\|_{0, \Omega}=1$ and

$$
a\left(\phi_{h}, v\right)=-\mu_{h}\left(\phi_{h}, v\right) \quad \forall v \in S^{h}(\Omega)
$$


which is equivalent to

$$
\tilde{a}\left(\phi_{h}, v\right)=\tilde{\mu}_{h}\left(\phi_{h}, v\right) \quad \forall v \in S^{h}(\Omega),
$$

with $\tilde{\mu}_{h}=-\mu_{h}+\xi$. In the following discussion, we assume that $\left(-\mu_{h}, \phi_{h}\right)$ and $\left(\tilde{\mu}_{h}, \phi_{h}\right)$ are the first eigenpair of (2.8) and (2.9), respectively.

Set

$$
\begin{aligned}
\rho_{\Omega}(h) & =\sup _{f \in L^{2}(\Omega),\|f\|_{0, \Omega}=1} \inf _{v \in S^{h}(\Omega)}\left\|(-\triangle-V(\cdot, \omega)+\xi)^{-1} f-v\right\|_{1, \Omega}, \\
\delta_{h}(-\mu) & =\sup _{u \in M(-\mu),\|u\|_{0, \Omega}=1} \inf _{v \in S^{h}(\Omega)}\|u-v\|_{1, \Omega}
\end{aligned}
$$

with

$$
M(-\mu)=\left\{w \in H^{1}(\Omega): w \text { is an eigenvector of }(2.2) \text { corresponding to }-\mu\right\} .
$$

For the standard finite element solution $\left(-\mu_{h}, \phi_{h}\right)$, the following estimates hold $[3,4$, $9,38]$.

THEOREM 2.2. There hold

$$
\begin{array}{r}
\left\|\phi-\phi_{h}\right\|_{1, \Omega} \leq C \delta_{h}(-\mu), \\
\left\|\phi-\phi_{h}\right\|_{0, \Omega} \leq C \rho_{\Omega}(h)\left\|\phi-\phi_{h}\right\|_{1, \Omega}, \\
-\mu \leq-\mu_{h} \leq-\mu+C \delta_{h}^{2}(-\mu),
\end{array}
$$

where $C$ is independent of the mesh parameter $h$. Moreover, if $\Omega$ is convex or a domain with a smooth boundary, then

$$
\mu-\mu_{h}+\left\|\phi-\phi_{h}\right\|_{0, \Omega}+h\left\|\phi-\phi_{h}\right\|_{1, \Omega} \leq C h^{2} .
$$

2.3. Two-scale discretization scheme. To reduce computational cost, let us present a two-scale scheme dating back to [23]; see also a general framework in [38], where $a(\cdot, \cdot)$ is a (deterministic) positive symmetric definite bilinear form. We extend the two-scale approach to solving (2.1).

Let $H \gg h$ and assume that $S^{H}(\Omega) \subset S^{h}(\Omega)$. We put the mesh size to the superscript and subscript of an eigenpair to tell the difference between the one-scale and two-scale finite element solution on the corresponding mesh; e.g., we use $\left(\mu_{H}, \phi_{H}\right)$ and $\left(\mu_{h}, \phi_{h}\right)$ to denote the one-scale solution on $T^{H}(\Omega)$ and $T^{h}(\Omega)$, respectively, and use $\left(\mu^{h}, \phi^{h}\right)$ to denote the two-scale solution associated with the fine mesh $T^{h}(\Omega)$. The two-scale finite element scheme for (2.3) is as follows.

Two-scale discretization scheme.

Step 1. Find $\left(\tilde{\mu}_{H}, \phi_{H}\right) \in \mathbb{R} \times S^{H}(\Omega)$ such that $\left\|\phi_{H}\right\|_{0, \Omega}=1$ and

$$
\tilde{a}\left(\phi_{H}, v\right)=\tilde{\mu}_{H}\left(\phi_{H}, v\right) \quad \forall v \in S^{H}(\Omega) .
$$

Step 2. Find $\phi^{h} \in S^{h}(\Omega)$ such that

$$
\int_{\Omega} \nabla \phi^{h} \nabla v+\xi_{0} \phi^{h} v=\tilde{\mu}_{H}\left(\phi_{H}, v\right)+\left(\left(V(y, \omega)-\xi+\xi_{0}\right) \phi_{H}, v\right) \quad \forall v \in S^{h}(\Omega),
$$

where $\xi_{0}$ is some (deterministic) positive constant. 
Step 3. Compute the Rayleigh quotient

$$
\tilde{\mu}^{h}=\frac{\tilde{a}\left(\phi^{h}, \phi^{h}\right)}{\left(\phi^{h}, \phi^{h}\right)}
$$

and set $\mu^{h}=-\tilde{\mu}^{h}+\xi$.

The error estimate of the two-scale solution reads as follows.

THEOREM 2.3. Let $(\mu, \phi)$ be the principal eigenpair of $(2.2)$ and $\left(\mu^{h}, \phi^{h}\right)$ be obtained from the two-scale scheme. Then

$$
\begin{aligned}
\left\|\phi-\phi^{h}\right\|_{1, \Omega} & \leq C\left(\delta_{H}^{2}(-\mu)+\rho(H) \delta_{H}(-\mu)+\delta_{h}(-\mu)\right), \\
\left|\mu-\mu^{h}\right| & \leq C\left(\delta_{H}^{4}(-\mu)+\rho^{2}(H) \delta_{H}^{2}(-\mu)+\delta_{h}^{2}(-\mu)\right) .
\end{aligned}
$$

Moreover, if $\Omega$ is convex or a domain with a smooth boundary, then for $h=\mathcal{O}\left(H^{2}\right)$ there holds

$$
\left|\mu-\mu^{h}\right|+h\left\|\phi-\phi^{h}\right\|_{1, \Omega} \leq C h^{2} .
$$

Proof. From the construction of $\phi^{h}$ and (2.8), we have

$$
\begin{aligned}
& \int_{\Omega} \nabla\left(\phi^{h}-\phi_{h}\right) \nabla v+\xi_{0}\left(\phi^{h}-\phi_{h}\right) v \\
= & \tilde{\mu}_{H}\left(\phi_{H}, v\right)-\tilde{\mu}_{h}\left(\phi_{h}, v\right)+\left(\left(V(y, \omega)-\xi+\xi_{0}\right)\left(\phi_{H}-\phi_{h}\right), v\right) \quad \forall v \in S^{h}(\Omega) .
\end{aligned}
$$

It follows from $V(y, \omega) \in L^{\infty}(\Omega)$ and the identity

$$
\tilde{\mu}_{H}\left(\phi_{H}, v\right)-\tilde{\mu}_{h}\left(\phi_{h}, v\right)=\left(\tilde{\mu}_{H}-\tilde{\mu}_{h}\right)\left(\phi_{H}, v\right)+\tilde{\mu}_{h}\left(\phi_{H}-\phi_{h}, v\right) \quad \forall v \in S^{h}(\Omega)
$$

that

$$
\left\|\phi^{h}-\phi_{h}\right\|_{1, \Omega} \leq C\left(\left|\mu_{h}-\mu_{H}\right|+\left\|\phi_{H}-\phi_{h}\right\|_{0, \Omega}\right),
$$

which together with the triangular inequality leads to

$$
\begin{aligned}
\left\|\phi^{h}-\phi\right\|_{1, \Omega} & \leq\left\|\phi^{h}-\phi_{h}\right\|_{1, \Omega}+\left\|\phi_{h}-\phi\right\|_{1, \Omega} \\
& \leq C\left(\left|\mu_{h}-\mu_{H}\right|+\left\|\phi_{H}-\phi_{h}\right\|_{0, \Omega}\right)+\left\|\phi_{h}-\phi\right\|_{1, \Omega} .
\end{aligned}
$$

Combining the triangular inequalities with Theorem 2.2 gives

$$
\begin{aligned}
\left|\mu_{h}-\mu_{H}\right| & \leq\left|\mu_{h}-\mu\right|+\left|\mu_{H}-\mu\right| \leq C \delta_{H}^{2}(-\mu), \\
\left\|\phi_{H}-\phi_{h}\right\|_{0, \Omega} & \leq\left\|\phi_{H}-\phi\right\|_{0, \Omega}+\left\|\phi_{h}-\phi\right\|_{0, \Omega} \leq C \rho(H) \delta_{H}(-\mu),
\end{aligned}
$$

which leads to (2.15). Proposition 2.1 and (2.15) imply (2.16). Theorem 2.2, (2.15), and (2.16) produce (2.17). This completes the proof.

Theorem 2.3 says that the resulting two-scale approximations of eigenvalue and eigenfunction still maintain optimal accuracy.

REMARK 2.1. In Step 2, the random term appears on the right-hand side only, and the fine mesh computation is on a boundary value problem. The eigenvalue problem is on a coarse mesh in Step 1 . If the absolute value of the random term $V(y, \omega)$ is bounded by some deterministic positive constant $v_{0}$, then $\xi$ in (2.9) (and hence Step 1) and $\xi_{0}$ in Step 2 can be selected to be $v_{0}$. 
REMARK 2.2. We may also obtain similar results for the following scheme (cf. [38]):

Step 1. Find $\left(\tilde{\mu}_{H}, \phi_{H}\right) \in \mathbb{R} \times S^{H}(\Omega)$ such that $\left\|\phi_{H}\right\|_{0, \Omega}=1$ and

$$
\tilde{a}\left(\phi_{H}, v\right)=\tilde{\mu}_{H}\left(\phi_{H}, v\right) \quad \forall v \in S^{H}(\Omega) .
$$

Step 2. Find $\phi^{h} \in S^{h}(\Omega)$ satisfying

$$
\tilde{a}\left(\phi^{h}, v\right)=\tilde{\mu}_{H}\left(\phi_{H}, v\right) \quad \forall v \in S^{h}(\Omega) .
$$

Step 3. Compute the Rayleigh quotient

$$
\tilde{\mu}^{h}=\frac{\tilde{a}\left(\phi^{h}, \phi^{h}\right)}{\left(\phi^{h}, \phi^{h}\right)}
$$

and set $\mu^{h}=-\tilde{\mu}^{h}+\xi$.

2.4. A thin domain theory. Consider the cross section $\Omega=[0, \epsilon] \times\left[0, \frac{1}{\epsilon}\right]$ for $\epsilon$ small. The area of $\Omega$ is 1 , while the aspect ratio is $\frac{1}{\epsilon^{2}}$. Integrating (1.3) over $y_{1}=[0, \epsilon]$ and applying the zero Neumann boundary condition gives

$$
\tilde{\phi}_{y_{2} y_{2}}+\left(\lambda^{2}+\lambda \tilde{b}\left(y_{2}, \omega\right)+f^{\prime}(0)\right) \tilde{\phi} \approx \mu \tilde{\phi},
$$

where $\tilde{\phi}\left(y_{2}\right)$ is the integral average of $\phi\left(y_{1}, y_{2}\right)$ over $y_{1} \in[0, \epsilon]$, and $\tilde{b}\left(y_{2}, \omega\right)$ is the integral average of $b\left(y_{1}, y_{2}, \omega\right)$ over $y_{1} \in[0, \epsilon]$. An approximation is made so that the integral average of $b\left(y_{1}, y_{2}, \omega\right) \phi$ is replaced to leading order by the product of the integral averages of the two factors. The eigenvalue problem (2.19) is on the large interval $\left[0, \frac{1}{\epsilon}\right]$. For a Gaussian process $\tilde{b}\left(y_{2}, \omega\right)$ with large enough root mean square amplitudes, the principal eigenvalue $\mu$ behaves like the running maximum of $\tilde{b}[26]$; the latter scales as $\sqrt{-2 \log \epsilon}$ in probability (Theorem 6.9.5 of Chapter 6 of [2]). Hence $\mu$ increases with the aspect ratio in probability, and it follows from (1.2) that the front speed also increases with the aspect ratio in this regime.

3. Numerical results. In this section, we report numerical results using both the standard finite element scheme and the two-scale finite element scheme.

For a given constant $\lambda>0$, we find $\mu(\lambda, \omega) \in \mathbb{R}$ such that

$$
(\nabla \phi, \nabla v)-\left(\left[\lambda^{2}+\lambda b(y, \omega)+f^{\prime}(0)\right] \phi, v\right)=-\mu(\lambda, \omega)(\phi, v) \quad \forall v \in H^{1}(\Omega),
$$

where $\Omega$ is a two-dimensional domain and $b(y, \omega)$ is a random process. Let $S^{h}(\Omega)$ be the linear finite element space over a uniform mesh $T^{h}(\Omega)$ with mesh size $h$.

The standard finite element scheme is the following: Find $\left(\mu_{h}, \phi_{h}\right) \in \mathbb{R} \times S^{h}(\Omega)$ such that $\left\|\phi_{h}\right\|_{0, \Omega}=1$ and

$$
\left(\nabla \phi_{h}, \nabla v\right)-\left(\left[\lambda^{2}+\lambda b(y, \omega)+f^{\prime}(0)\right] \phi_{h}, v\right)=-\mu_{h}(\lambda, \omega)\left(\phi_{h}, v\right) \quad \forall v \in S^{h}(\Omega) .
$$

To approximate the principal eigenvalue, we apply the inverse power method to solve the above algebraic system.

To generate the random process $b(y, \omega)$, we adopt the random Fourier method [21]

$$
\begin{aligned}
b_{\text {Four }}(y)= & \sum_{j_{1}=0}^{M} \sum_{j_{2}=0}^{M} \exp \left(-\left(\left(j_{1} d k_{1}\right)^{2}+\left(j_{2} d k_{2}\right)^{2}\right) / 2\right) \sqrt{d k_{1}^{2}+d k_{2}^{2}} \\
& \cdot\left[\zeta_{j_{1} j_{2}} \cos 2 \pi\left(j_{1} d k_{1}, j_{2} d k_{2}\right) \cdot y+\eta_{j_{1} j_{2}} \sin 2 \pi\left(j_{1} d k_{1}, j_{2} d k_{2}\right) \cdot y\right],
\end{aligned}
$$




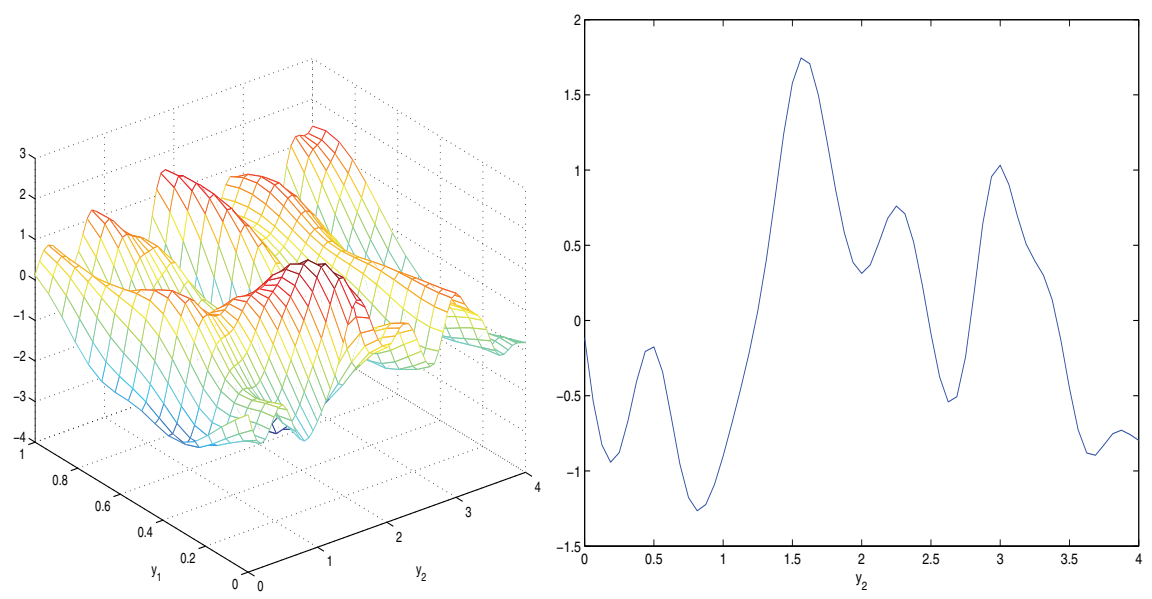

FIG. 1. A realization of random process $b_{F o u r}(y)$ on the $\left(y_{1}, y_{2}\right) \in[0,1] \times[0,4]$ square domain (left) and on the axis $\left(0, y_{2}\right) \in[0,4]$. The grid size is $1 / 16$.

where $y=\left(y_{1}, y_{2}\right)^{T} \in \Omega, d k_{1}$ and $d k_{2}$ are wave number spacing, and $M$ is the highest Fourier mode retained. $\left\{\zeta_{j_{1} j_{2}}\right\}_{j_{1}, j_{2}=1}^{M}$ and $\left\{\eta_{j_{1} j_{2}}\right\}_{j_{1}, j_{2}=1}^{M}$ are independent standard unit Gaussian random variables. The energy spectrum of the process has exponential decay towards high frequencies. The random field $b$ is also statistically isotropic. Figure 1 shows a realization of the random field at grid size $1 / 16$.

Consider the scaled realizations $\delta b\left(y, \omega_{i}\right)(i=1, \ldots, N), \delta$ being a positive deterministic constant. We first solve (3.2) and obtain an approximation of the principal eigenvalue $\mu\left(\lambda, \omega_{i}\right)$. Then we find a sample of minimal speed

$$
c_{i}^{*}(\delta)=\inf _{\lambda>0} \frac{\mu\left(\lambda, \omega_{i}\right)}{\lambda}
$$

and the average

$$
E\left[c^{*}(\delta)\right] \approx \bar{E}=c_{0}^{*}+\frac{1}{N} \sum_{i=1}^{N} M_{i}(\delta)
$$

where $c_{0}^{*}=2 \sqrt{f^{\prime}(0)}$ denotes the minimal speed in the case of zero advection, $M_{i}(\delta)=$ $c_{i}^{*}(\delta)-c_{0}^{*}-\delta \bar{b}_{i}$, and $\bar{b}_{i}$ is the integral average of $b\left(y, \omega_{i}\right)$ over cross section $\Omega$. Subtracting $\delta \bar{b}_{i}$ improves the accuracy of finite sample approximation of expectation [26, 28].

The most expensive part of the computation is solving linear eigenvalue problem (3.1). Due to the large number of such eigenvalue problems involved (on the scale of $10^{4}$ ), the two-scale method is very helpful for reducing computational time without losing accuracy.

We will solve (3.1) both for square and elliptical domains. We first show fully resolved computational results from the one-scale method (the standard finite element method) on the effect of domain sizes. Later, we compare these as a benchmark with the results of the two-scale scheme to demonstrate the advantage of the latter. In the two-scale method, we choose the coarse mesh size $H=1 / 4$ and the fine mesh size $h=1 / 16$.

3.1. Resolved computation of one-scale method. First, we consider the square domains. We use the standard finite element method to compute the average 

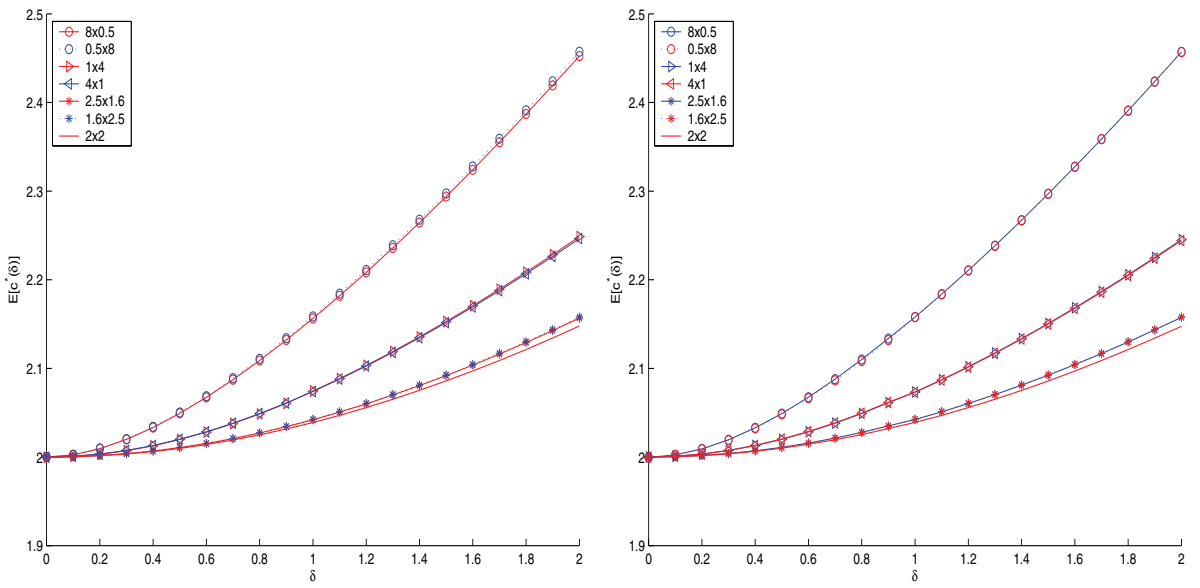

FIG. 2. The average enhancement of minimal speed as a function of $\delta \in[0,2]$. Left (right) panel is with $N=2000$ (10000) samples and mesh size $h=1 / 16$. The legends show that the different lines refer to different domain aspect ratios.
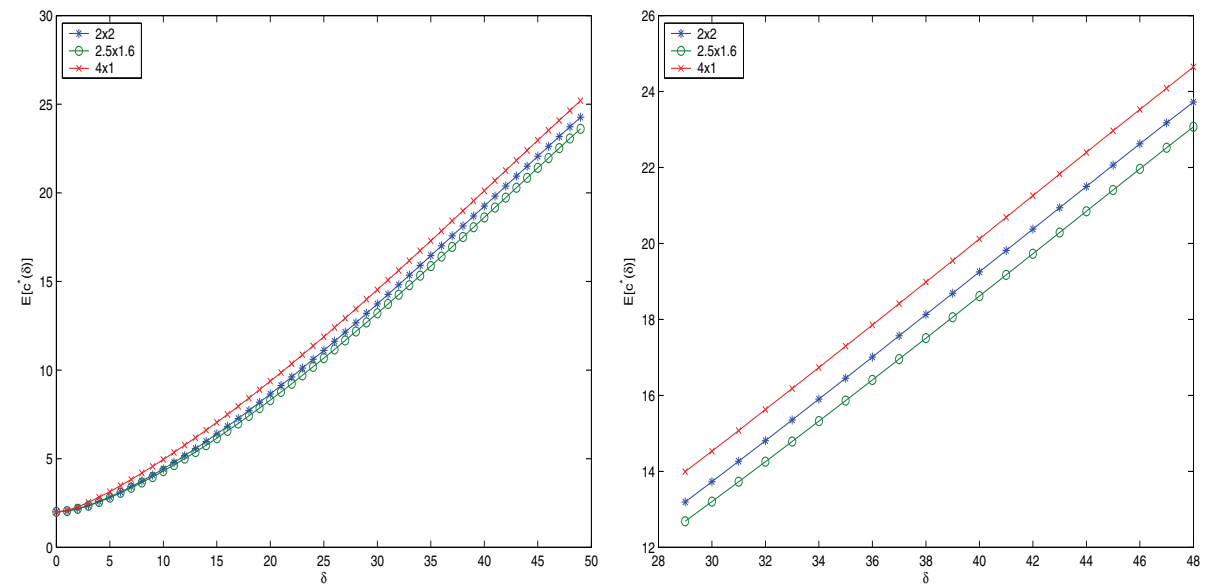

FIG. 3. Average enhancement of minimal speed with 1000 samples. The left figure shows the values when $\delta \in[0,50]$, and the right figure shows the values when $\delta \in[30,50]$. The legends show that the different lines refer to different domain aspect ratios.

speed with various domain aspect ratios. Figure 2 plots the ensemble averaged front speeds as a function of scaling parameter $\delta \in[0,2]$ when the domains have different aspect ratios but the same area (equal to 4$)$. Comparing the left panel $(N=2000)$ and the right panel $(N=10000)$ shows that convergence in $N$ for averaged speeds occurs at $N=2000$, in fact beginning even at $N=1000$. We observe that as the domain aspect ratio increases, so does the average front speed. Because the random field $b$ is isotropic, the average speed is invariant as the domain dimensions in $y_{1}$ and $y_{2}$ switch. The monotonicity of the plotted curves comes from the enhancement of front speeds by shear flows (see [28] and the references therein).

Figure 3 plots the enhancement of the minimal speed in the range $\delta \in[0,50]$. The figure recovers the fact that when $\delta$ is small (large), the enhancement obeys a quadratic (linear) law in $\delta$. Moreover, it shows that the effect of aspect ratio on 

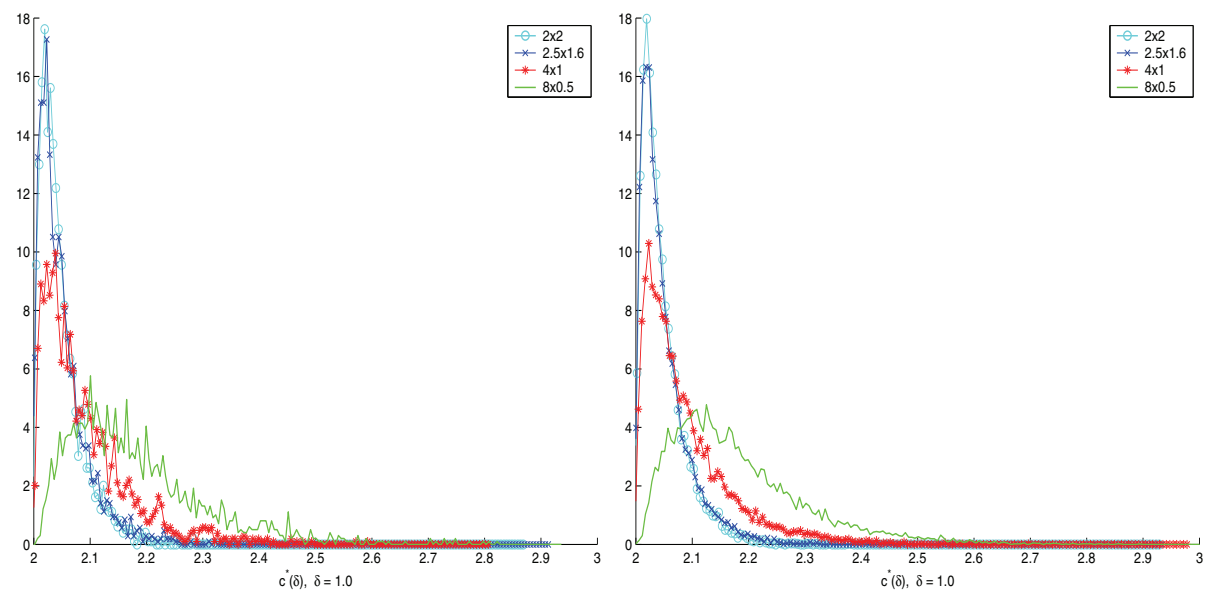

FIG. 4. Probability distribution functions of enhancement $M(\delta)$ at $\delta=1.0$ with $N=2000$ samples (left) and $N=10000$ samples (right) for rectangular domains at four aspect ratios.

average front speeds persists to larger values of $\delta$. We omitted plotting the $8 \times 0.5$ curve partly because the $2 \times 2$ and $2.5 \times 1.6$ curves would have looked too close.

We also compute the distributions of the speed enhancement $M(\delta)$ at fixed $\delta$. We partition the range of $M$ values into $Q=300$ bins and approximate the speed distribution as

$$
\operatorname{pdf}(x)=\frac{1}{N} \sum_{i=1}^{N} \frac{\chi_{j}\left(M_{i}(\delta)\right)}{\left(x_{j+1}-x_{j}\right)} \quad \text { if } x \in\left[x_{j}, x_{j+1}\right), \quad j=1, \ldots, Q,
$$

where $\chi_{j}(x)$ is the characteristic function of the interval $\left[x_{j}, x_{j+1}\right)$. Figure 4 shows the values of pdf at various domain aspect ratios with $N=2000$ and 10000 samples at $\delta=1$. Both the mean and variance of the speed enhancement increase with the aspect ratio.

Next we compute the front speeds in the case of elliptical domains. The boundary equation of the elliptical domain is

$$
\frac{x^{2}}{a^{2} / \pi}+\frac{y^{2}}{b^{2} / \pi}=1
$$

where $a$ and $b$ are positive parameters controlling the aspect ratio and area. Figure 5 shows that the average enhancement of minimal speed increases with the domain area at the same aspect ratio.

Figure 6 shows the values of $E\left[c^{*}\right]$ when $0 \leq \delta \leq 2$, and Figure 7 shows the values when $0 \leq \delta<50$. The legends of these figures show the values of $a$ and $b$ and the number of samples.

3.2. Computations by two-scale method. Using the two-scale scheme, we computed the average enhancement of minimal speed with $N=1000$ samples both in square and elliptical domains. Choose $H=1 / 4$ and $h=1 / 16$ to keep $h=H^{2}$. In Figure 8, the fine-scale corrections of Steps 2 and 3 of the method clearly make an appreciable difference in averaged speeds.

We see in Figure 8 that the average enhancement of minimal speed has little difference whether the domain is square shape or elliptical, as long as the area and 


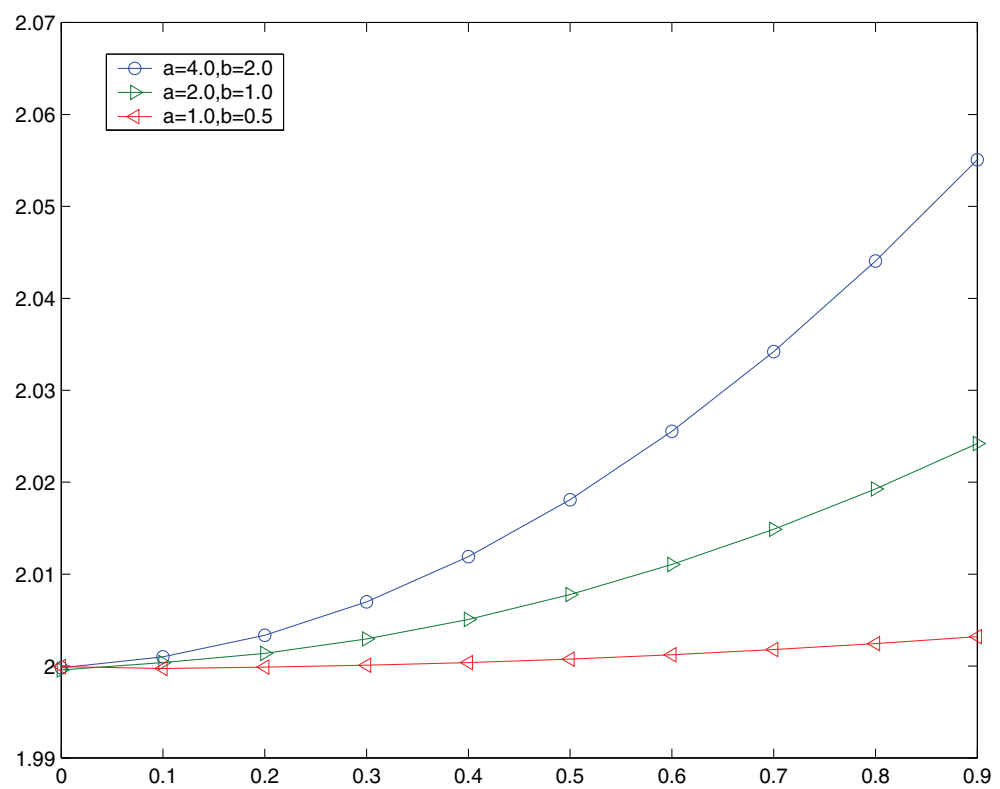

FIG. 5. The values of $E\left[c^{*}\right]$ over elliptical domains of different areas and the same aspect ratio with $N=1000$ samples.

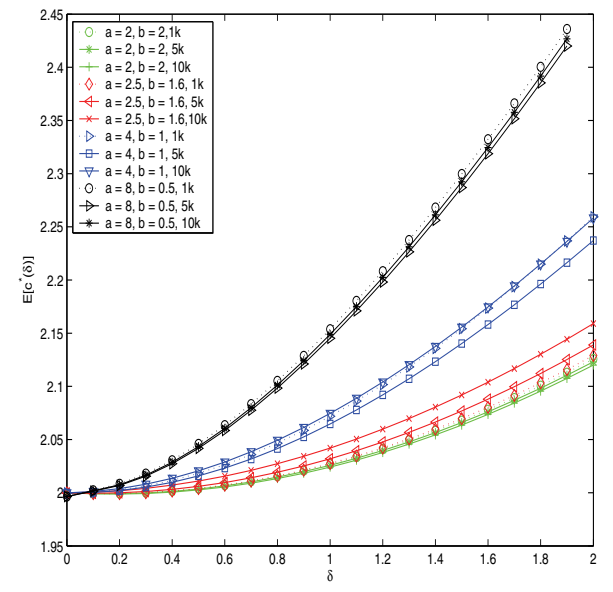

FIG. 6. The values of $E\left[c^{*}\right]$ with different domain aspect ratios and the same area at $\delta \in[0,2]$ with $N=1000$, 5000, and 10000 samples.

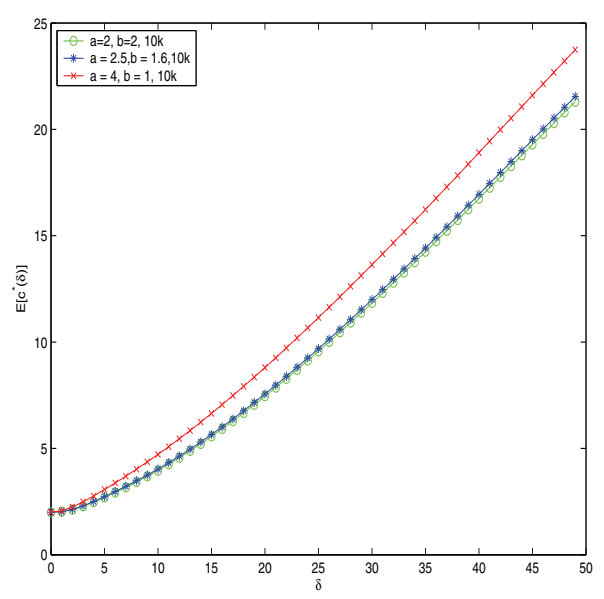

FIG. 7. The values of $E\left[c^{*}\right]$ with different domain aspect ratios and the same area at $\delta \in$ $[0,50]$ with $N=10000$ samples.

the aspect ratio are the same. Figure 9 shows that results of the two-scale scheme agree quite well with those from the standard (one-scale) finite element discretization. Figure 10 shows that the two-scale scheme saves computing time significantly when compared with the standard finite element method. Here we use the same numerical linear algebra solver when implementing the two discretization schemes. We see in Figure 10 that the $8.0 \times 0.5$ square domain takes much more computing time. The reason is that, for such a thin domain, the matrix condition gets worse, and so the 

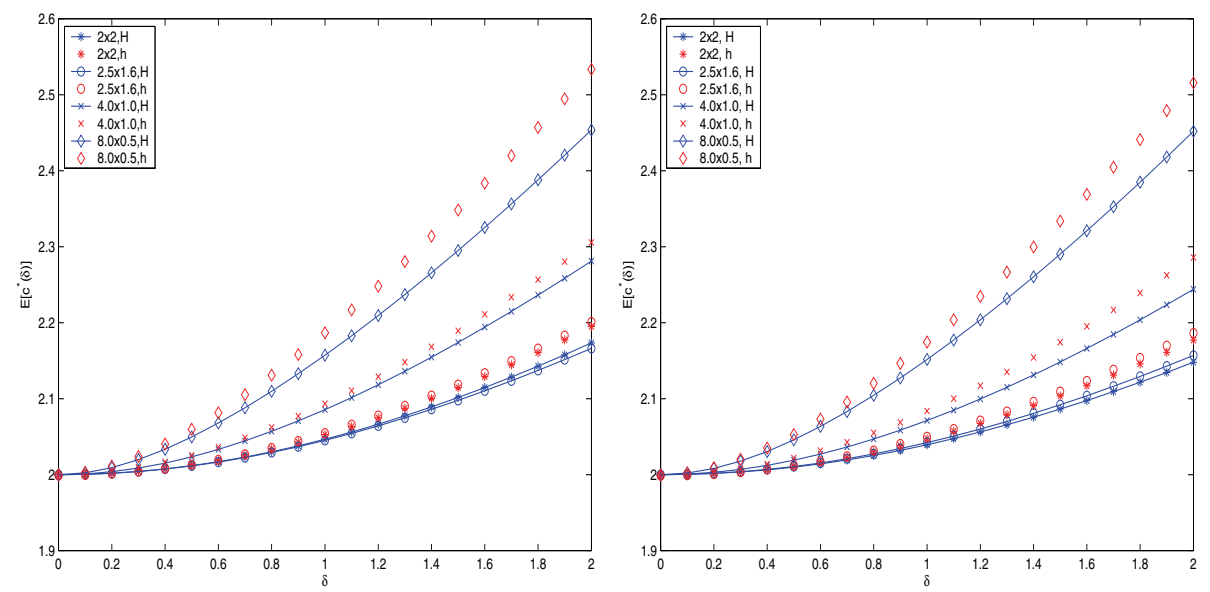

FIG. 8. The average enhancement of minimal speed with $N=1000$ samples, computed by the two-scale scheme. The left panel shows the enhancement of speed in the coarse mesh (results by Step 1 in the two-scale scheme) and after the fine mesh correction (results by Steps 2 and 3 in the two-scale scheme) for the square domain. The right panel is for the elliptical domain.

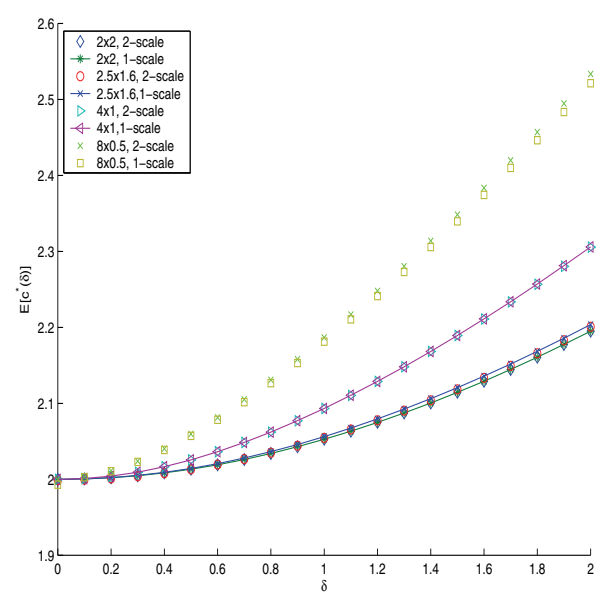

FIG. 9. The average enhancement of minimal speed computed with the fine mesh $h=1 / 16$. The data points from the two-scale method come close to those from the standard finite element method.

convergence rate is slower when using the inverse power method to solve the eigenvalue problem.

4. Concluding remarks. Based on the variational principle of KPP front speeds in random shear flows in cylinders, we carried out finite element computations of KPP front speed ensemble by using both the one-scale finite element discretization and the two-scale finite element discretization. The numerically computed average speed enhancement is in agreement with theoretical analysis. In particular, equal area domains with larger aspect ratios increase the mean and variance of the front speeds in the parameter regimes simulated. The two-scale discretization scheme is much more efficient than the one-scale discretization while achieving the same accuracy. In future work, we plan to study front speed ensemble in 


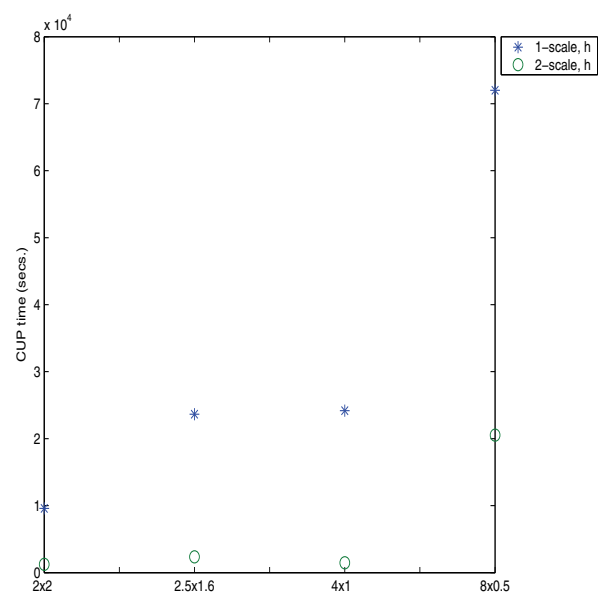

FIG. 10. The time used to produce the data in Figure 9 by one-scale and two-scale methods.

space-time random flows $[29,31]$ by extending the two-scale method to parabolic problems.

Acknowledgments. The authors wish to thank Professor S. Shu at Xiangtan University for providing the grid generation software for elliptical domains and the anonymous referees for their helpful suggestions.

\section{REFERENCES}

[1] R. A. Adams, Sobolev Spaces, Academic Press, New York, 1975.

[2] R. AdLer, The Geometry of Random Fields, John Wiley and Sons, Chichester, UK, 1980.

[3] I. BABUska AND J. E. Osborn, Finite element-Galerkin approximation of the eigenvalues and eigenvectors of selfadjoint problems, Math. Comp., 52 (1989), pp. 275-297.

[4] I. Babuska and J. E. Osborn, Eigenvalue problems, in Handbook of Numerical Analysis, Volume II. Finite Element Methods (Part 1), P. G. Ciarlet and J. L. Lions, eds., NorthHolland, Amsterdam, 1991, pp. 641-787.

[5] H. Berestycki, F. Hamel, and N. Nadirashvili, Elliptic eigenvalue problems with large drift and applications to nonlinear propagation phenomena, Comm. Math. Phys., 253 (2005), pp. 451-480.

[6] H. Berestycki and L. Nirenberg, Travelling fronts in cylinders, Ann. Inst. H. Poincaré Anal. Non Linéaire, 9 (1992), pp. 497-572.

[7] A. Bourlioux And B. KhOuider, A rigorous asymptotic perspective on the large scale simulations of turbulent premixed flames, Multiscale Model. Simul., 6 (2007), pp. 287-307.

[8] S.-L. Chang, C.-S. Chien, And B.-W. Jeng, An efficient algorithm for the SchrödingerPoisson eigenvalue problem, J. Comput. Appl. Math., 205 (2007), pp. 509-532.

[9] F. Chatelin, Spectral Approximations of Linear Operators, Academic Press, New York, 1983.

[10] M. Chertkov And V. Yaкhot, Propagation of a Huygens front through turbulent medium, Phys. Rev. Lett., 80 (1998), pp. 2837-2840.

[11] C.-S. Chien And B.-W. Jeng, A two-grid discretization scheme for semilinear elliptic eigenvalue problems, SIAM J. Sci. Comput., 27 (2006), pp. 1287-1304.

[12] P. G. Ciarlet, The Finite Element Method for Elliptic Problems, North-Holland, Amsterdam, New York, Oxford, 1978.

[13] P. G. Ciarlet and J. L. Lions, eds., Handbook of Numerical Analysis, Volume II. Finite Element Methods (Part 1), North-Holland, Amsterdam, 1991.

[14] P. Clavin and F. Williams, Theory of premixed-flame propagation in large-scale turbulence, J. Fluid Mech., 90 (1979), pp. 598-604.

[15] P. Constantin, A. Kiselev, A. Oberman, and L. Ryzhik, Bulk burning rate in passivereactive diffusion, Arch. Ration. Mech. Anal., 154 (2000), pp. 53-91. 
[16] J. GÄrTner And M. FreidLin, The propagation of concentration waves in periodic and random media, Dokl. Akad. Nauk SSSR, 249 (1979), pp. 521-525.

[17] F. Hamel, Formules min-max pour les vitesses d'ondes progressives multidimensionnelles, Ann. Fac. Sci. Toulouse Math. (6), 8 (1999), pp. 259-280.

[18] S. Heinze, G. Papanicolaou, and A. Stevens, Variational principles for propagation speeds in inhomogeneous media, SIAM J. Appl. Math., 62 (2001), pp. 129-148.

[19] A. R. Kerstein And W. T. Ashurst, Propagation rate of growing interfaces in stirred fluids, Phys. Rev. Lett., 68 (1992), pp. 934-937.

[20] K. Kolman, A two-level method for nonsymmetric eigenvalue problems, Acta Math. Appl. Sin. Engl. Ser., 21 (2005), pp. 1-12.

[21] P. R. Kramer, A review of some Monte Carlo simulation methods for turbulent systems, Monte Carlo Methods Appl., 7 (2001), pp. 229-243.

[22] Q. LIN, Some problems concerning approximate solutions of operator equations, Acta Math. Sinica, 22 (1979), pp. 219-230 (in Chinese).

[23] Q. Lin And G. XIE, Accelerating the finite element method in eigenvalue problems, Kexue Tongbao (Chinese), 26 (1981), pp. 449-452.

[24] F. LiU AND A. Zhou, Two-scale finite element discretizations for parallel differential equations, J. Comput. Math., 24 (2006), pp. 373-392.

[25] A. Majda And P. Souganidis, Flame fronts in a turbulent combustion model with fractal velocity fields, Comm. Pure Appl. Math., 51 (1998), pp. 1337-1348.

[26] J. Nolen AND J. Xin, Variational principle based computation of KPP average front speeds in random shear flows, Methods Appl. Anal., 11 (2004), pp. 389-398.

[27] J. Nolen And J. Xin, Min-max variational principle and front speeds in random shear flows, Methods Appl. Anal., 11 (2004), pp. 635-644.

[28] J. Nolen And J. Xin, A variational principle based study of KPP minimal front speeds in random shears, Nonlinearity, 18 (2005), pp. 1655-1675.

[29] J. Nolen And J. XIN, Variational principle of KPP front speeds in temporally random shear flows with applications, Comm. Math. Phys., 269 (2007), pp. 493-532.

[30] J. Nolen And J. Xin, Computing reactive front speeds in random flows by variational principle, Phys. D, 237 (2008), pp. 3172-3177.

[31] J. Nolen AND J. XIN, Asymptotic spreading of KPP reactive fronts in incompressible spacetime random flows, Ann Inst. H. Poincaré Anal. Non Linéaire, to appear.

[32] P. Ronney, Some open issues in premixed turbulent combustion, in Modeling in Combustion Science, Lecture Notes in Phys. 449, J. D. Buckmaster and T. Takeno, eds., SpringerVerlag, Berlin, 1995, pp. 3-22.

[33] P. Ronney, B. Haslam, and N. Rhys, Front propagation rates in randomly stirred media, Phys. Rev. Lett., 74 (1995), pp. 3804-3807.

[34] L. Shen, Parallel Adaptive Finite Element Algorithms for Electronic Structure Computing Based on Density Functional Theory, Ph.D. thesis, Academy of Mathematics and Systems Science, Chinese Academy of Sciences, Beijing, China, 2005.

[35] I. H. SlOAN, Iterated Galerkin method for eigenvalue problems, SIAM J. Numer. Anal., 13 (1976), pp. 753-760.

[36] J. XIN, Front propagation in heterogeneous media, SIAM Rev., 42 (2000), pp. 161-230.

[37] J. XIN, KPP front speeds in random shears and the parabolic Anderson problem, Methods Appl. Anal., 10 (2003), pp. 191-198.

[38] J. Xu And A. Zhou, A two-grid discretization scheme for eigenvalue problems, Math. Comp., 70 (2001), pp. 17-25.

[39] V. Yакнот, Propagation velocity of premixed turbulent flames, Combust. Sci. Technol., 60 (1988), pp. 191-214

[40] A. Zlatos, Pulsating front speed-up and quenching of reaction by fast reaction, Nonlinearity, 20 (2007), pp. 2907-2921. 